\title{
Relationship between the Degree of Endoscopic Atrophy of the Gastric Mucosa and Carcinogenic Risk
}

\author{
Hironori Masuyama ${ }^{a}$ b Naoto Yoshitake $^{b}$ Takako Sasai $^{b}$ Tetsuya Nakamurab \\ Atsushi Masuyama $^{a}$ Toru Zuikic Kentaro Kurashina ${ }^{c}$ Mitsuyo Mieda ${ }^{d}$ Keijiro Sunada ${ }^{d}$ \\ Hironori Yamamoto $^{d}$ Kazutomo Togashi $^{\mathrm{e}}$ Akira Terano $^{\mathrm{b}}$ Hideyuki Hiraishi $^{\mathrm{b}}$ \\ a Masuyama Gastrointestinal Clinic, Otawara, 'b Department of Gastroenterology, Dokkyo Medical University, Mibu, \\ 'Department of Surgery, and d Division of Gastroenterology, Department of Medicine, Jichi Medical University, \\ Shimotsuke, and 'Department of Coloproctology, Aizu Medical Center, Fukushima Medical University, \\ Fukushima, Japan
}

\section{Key Words}

Gastric mucosal atrophy · Gastric cancer - Carcinogenesis .

Helicobacter pylori

\begin{abstract}
Background: The relationship between Helicobacter pylori infection and gastric cancer has been demonstrated, and the risk of gastric cancer occurrence is known to increase with the progression of atrophic changes associated with chronic gastritis. Endoscopic evaluation of the degree and extent of atrophy of the gastric mucosa is a simple and very important means of identifying a group at high risk for gastric cancer. This study aimed to clarify the carcinogenic risk in relation to the degree of atrophy. Methods: A total of 27,777 patients (272 with early gastric cancer and 135 with advanced gastric cancer) were included in this study. Endoscopically evaluated atrophy of the gastric mucosa was classified as C-0 to O-3 according to the Kimura and Takemoto classification system. Results: The cancer detection rate in relation to the degree of gastric mucosal atrophy was $0.04 \%$ (2/4,183 patients) for C-0, $0 \%(0 / 4,506)$ for C-1, $0.25 \%(9 / 3,660)$ for C-2, $0.71 \%$ $(21 / 2,960)$ for C-3, 1.32\% (75/5,684) for O-1,3.70\% $(140 / 3,780)$ for $\mathrm{O}-2$ and $5.33 \%(160 / 3,004)$ for $\mathrm{O}-3$. As to the proportions
\end{abstract}

\section{KARGER 125}

E-Mail karger@karger.com www.karger.com/dig
(C) 2015 The Author(s)

Published by S. Karger AG, Basel 0012-2823/15/0911-0030\$39.50/0

This article is licensed under the Creative Commons AttributionNonCommercial-NoDerivatives 4.0 International License (CC BYNC-ND) (http://www.karger.com/Services/OpenAccessLicense) Usage and distribution for commercial purposes as well as any distribution of modified material requires written permission. of differentiated and undifferentiated cancers, the latter were relatively frequent in the $\mathrm{C}-0$ to $\mathrm{C}-2$ groups, but differentiated cancers became predominant as atrophy progressed. On the other hand, the number of both differentiated and undifferentiated cancers detected increased as gastric mucosal atrophy progressed. In addition, open-type atrophy was found in 29 (96.7\%) of 30 patients with synchronous multiple gastric cancers and in all 20 patients with metachronous multiple gastric cancers. Conclusion: Endoscopic evaluation of gastric mucosal atrophy can provide a simple and reliable predictive index for both current and future carcinogenic risk.

(C) 2015 The Author(s)

Published by S. Karger AG, Basel

\section{Introduction}

The presence of atrophic gastritis in the background of gastric carcinogenesis has long been widely recognized, and it has been pointed out that atrophic gastritis represents a high risk for developing gastric cancer $[1,2]$. Until the discovery of Helicobacter pylori, aging and foods that damage the gastric mucosa had been considered to be causative factors for gastric mucosal atrophy. Recently, 
$H$. pylori was suggested to be the leading cause of gastric mucosal atrophy [3], and epidemiological research has led to the recognition that $H$. pylori infection, atrophic gastritis and gastric cancer are closely related $[4,5]$.

Infection with $H$. pylori is known to cause gastric mucosal atrophy and intestinal metaplasia, and the risk of gastric carcinogenesis increases along with the progression of these changes [6]. Therefore, it is very important to evaluate the degree and extent of gastric mucosal atrophy when identifying patients at high risk for developing gastric cancer. Although estimation of gastric cancer risk based on endoscopic findings has previously been attempted using gastric mucosal atrophy and intestinal metaplasia as indices [6], there have been no reports addressing carcinogenic risk in relation to precise classification of the degree of atrophy. It is true that routine observation has limitations when evaluating intestinal metaplasia based on endoscopic findings. Thus, we focused on endoscopic atrophic gastritis, which is observable by a routine simple method requiring no special devices or stains, and evaluated carcinogenic risk based on the precisely classified degree of atrophy.

\section{Methods}

\section{Patients}

Among a total of 32,854 patients who underwent endoscopic examination of the upper gastrointestinal tract at our institution during the 13 years between January 2001 and December 2013, 27,777 patients (272 with early gastric cancer, 135 with advanced gastric cancer) were included in this study. We excluded 4,373 patients who were examined after H. pylori eradication and 704 who had a remnant stomach. The study patients included 12,305 (44.3\%) men and 15,472 (55.7\%) women, with a mean age of 57.3 years; there was no age difference between the male and female patients. Written informed consent was obtained from all patients prior to the procedure.

\section{Evaluation of Gastric Mucosal Atrophy}

Endoscopic findings of gastric mucosal atrophy obtained by routine observation were classified according to the Kimura and Takemoto classification system into the closed type (C-1 to C-3), characterized by borders of atrophy not exceeding the cardia on the lesser curvature side of the gastric corpus, and the open type (O-1 to O-3), characterized by borders of atrophy exceeding the cardia into the greater curvature side [7]. Characteristic features of each type are as follows: atrophic mucosa is limited to the antrum in $\mathrm{C}-1$; limited to the gastric angle or the lower corpus in C-2; limited to the upper corpus in $\mathrm{C} 3$, and limited to the surroundings of the gastric cardia, with maintained folds of the greater curvature, in O-1; atrophy is present in the entire stomach, with lack of folds in the greater curvature as a whole in $\mathrm{O}-3$, and $\mathrm{O}-2$ is an intermediate type between O-1 and O-3. Atrophy is absent in C-0. All endoscopic findings were evaluated by a single principal investigator.

Degree of Endoscopic Atrophy of Gastric Mucosa and Carcinogenic Risk
Diagnosis of Gastric Cancer

Gastric cancer was diagnosed based not only on endoscopic findings but also biopsy or subsequent pathological findings of resected specimens according to the Japanese Classification of Gastric Carcinoma [8]. Papillary adenocarcinoma and tubular adenocarcinoma were classified as differentiated type, whereas poorly differentiated adenocarcinoma, signet-ring cell carcinoma and mucinous adenocarcinoma were classified as undifferentiated type (the intestinal type and diffuse type of the Lauren classification commonly used in Europe and the USA are essentially synonymous with differentiated type and undifferentiated type, respectively [9]). When different histological types were intermingled within a tumor, the quantitatively dominant type was adopted. Metachronous cancer was defined as a new lesion occurring in another part of the stomach more than 1 year after the initial lesion had been treated.

\section{Statistical Analysis}

Univariate analysis was carried out for each item. Student's t test was used for the analysis of age. Although other data were analyzed employing the $\chi^{2}$ test, Fisher's exact probability test was used for items that had an expected value of 5 or less. The level of statistical significance was $\mathrm{p}<0.05$.

\section{Results}

Among 407 patients in whom gastric cancer was detected, there were 288 (70.8\%) men and 119 (29.2\%) women; gastric cancer was significantly more frequent in men than women $(\mathrm{p}<0.001)$. There was no significant difference in mean age between men and women.

The gastric cancer detection rate in relation to the degree of gastric mucosal atrophy was $0.04 \%$, i.e. 2 ( 1 early, 1 advanced) out of 4,183 patients had C-0 atrophy, $0 \%$ $(0 / 4,506)$ had C-1 atrophy, $0.25 \%(9 / 3,660,7$ early, 2 advanced) had C-2 atrophy, $0.71 \%(21 / 2,960,14$ early, 7 advanced) had C-3 atrophy, 1.32\% (75/5,684, 47 early, 28 advanced 28) had O-1 atrophy, 3.70\% (140/3,780, 96 early, 44 advanced) had O-2 atrophy and 5.33\% (160/3,004, 107 early, 53 advanced) had O-3 atrophy. The gastric cancer detection rate increased significantly with the progression of gastric mucosal atrophy (fig. 1).

The gender and age of the total cases and early gastric cancer cases in the C-0 to O-3 groups are shown in table 1 . Patient age and the proportion of male patients increased with the progression of gastric mucosal atrophy. In addition, age was greater amongst early gastric cancer cases compared with total cases, and early gastric cancer was more frequent in men than women in each group (C-2 to O-3 groups).

Among the early gastric cancers detected, there were 220 cases with differentiated tumors, 50 with undifferen- 
Fig. 1. The gastric cancer detection rate in relation to the degree of gastric mucosal atrophy: the rate increased significantly with the progression of gastric mucosal atrophy. All data are percentages and the table displays the $\mathrm{p}$ values.

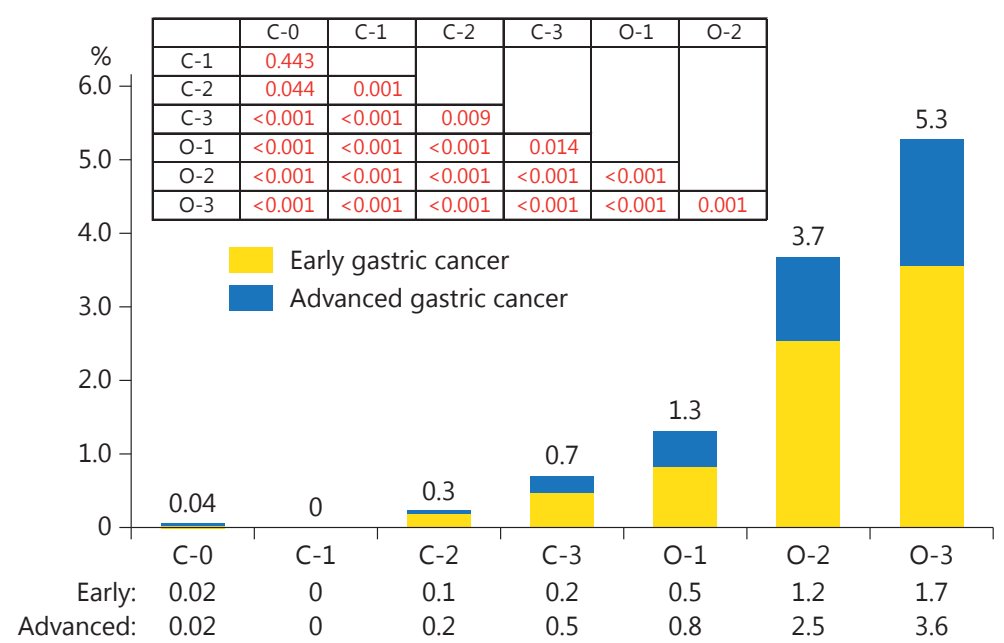

Table 1. Gender and age of total cases and early gastric cancer cases in the C-0 to O-3 groups

\begin{tabular}{llllllll}
\hline & C-0 & C-1 & C-2 & C-3 & O-1 & O-2 & O-3 \\
\hline Total & & & & & & \\
Cases & 4,183 & 4,506 & 3,660 & 2,960 & 5,684 & 3,780 & 3,004 \\
Male & $1,355(32.4)$ & $1,793(39.8)$ & $1,676(45.8)$ & $1,205(40.7)$ & $2,588(45.5)$ & $1,952(51.6)$ & $1,713(57.0)$ \\
Female & $2,828(67.6)$ & $2,713(60.2)$ & $1,984(54.2)$ & $1,755(59.3)$ & $3,096(54.5)$ & $1,828(48.4)$ & $1,291(43.0)$ \\
Age, years & $49.6 \pm 13.9$ & $52.5 \pm 14.7$ & $53.7 \pm 13.9$ & $54.8 \pm 13.0$ & $59.4 \pm 11.5$ & $64.0 \pm 10.9$ & $69.0 \pm 10.4$ \\
\hline Early gastric cancer & & & & & & \\
Cases & 1 & 0 & 7 & & & \\
Male & $1(100)$ & 0 & $3(42.9)$ & $7(50.0)$ & $35(74.5)$ & $71(74.0)$ & $79(73.8)$ \\
Female & 0 & 0 & $4(57.1)$ & $7(50.0)$ & $12(25.5)$ & $25(26.0)$ & $28(26.2)$ \\
Age, years & 39.0 & & $63.4 \pm 11.5$ & $56.8 \pm 11.5$ & $61.0 \pm 11.5$ & $65.4 \pm 11.6$ & $70.9 \pm 11.6$ \\
\hline
\end{tabular}

Values are $\mathrm{n}(\%)$ or mean \pm SD.

tiated tumors and 2 with an unclear histological type. Patients with differentiated cancers comprised 158 (71.8\%) men and $62(28.2 \%)$ women, with mean ages of 67.0 and 70.8 years, respectively. Among those with undifferentiated cancers, there were $34(68.0 \%)$ men and $16(32.0 \%)$ women, with mean ages of 58.3 and 58.0 years, respectively. Both differentiated and undifferentiated tumors were more frequent in men than women $(\mathrm{p}<0.001$ for both types of cancer), and in younger patients undifferentiated cancers were significantly more frequent than differentiated cancers $(\mathrm{p}<0.001)$.
When the proportions of undifferentiated and differentiated cancers were determined in relation to the degree of atrophy, there were 3 (37.5\%) cases with undifferentiated tumors and $5(62.5 \%)$ with differentiated tumors in the C-0 to C-2 groups, 16 (25.4\%) with undifferentiated tumors and $47(74.6 \%)$ with differentiated tumors in the C-3 to O-1 groups, and 32 (15.6\%) with undifferentiated tumors and 173 (84.4\%) with differentiated tumors in the O-2 to O-3 groups (fig. 2). Regarding the proportions of differentiated and undifferentiated cancers, undifferentiated cancers were relatively frequent when gas- 


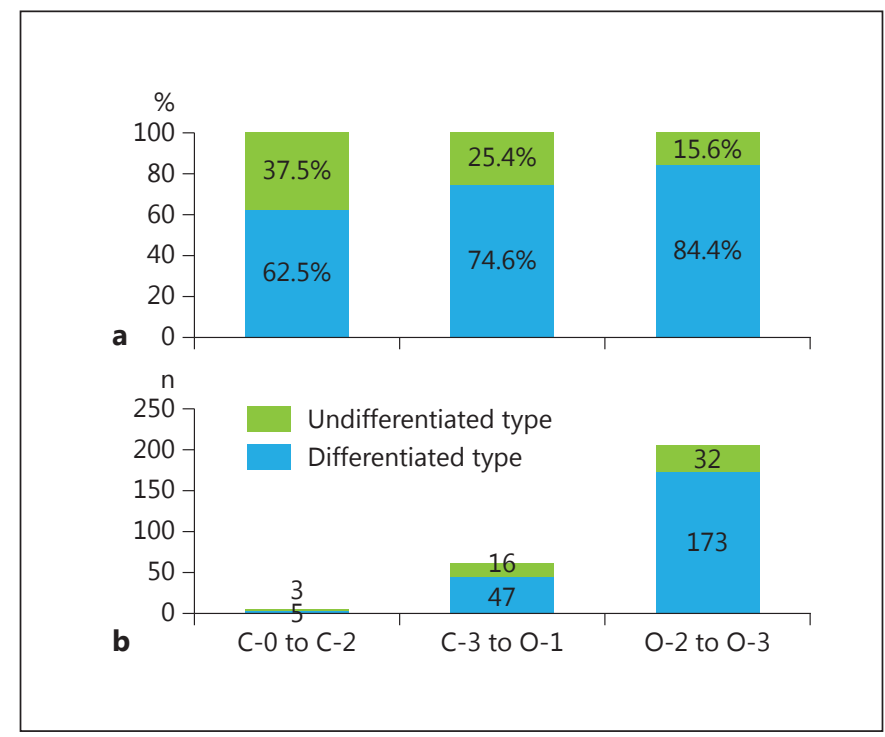

Fig. 2. The proportion (a) and number (b) of undifferentiated and differentiated gastric cancers in relation to the degree of gastric mucosal atrophy. Undifferentiated cancers were relatively frequent when gastric mucosal atrophy was absent or mild, but differentiated cancers tended to become predominant along with the progression of gastric mucosal atrophy. On the other hand, the number of both differentiated and undifferentiated cancers detected increased as the degree of gastric mucosal atrophy progressed.

tric mucosal atrophy was absent or mild, but differentiated cancers tended to become predominant along with the progression of gastric mucosal atrophy $(\mathrm{C}-0$ to $\mathrm{C}-2 \mathrm{vs}$. $\mathrm{C}-3$ to $\mathrm{O}-1$ groups, $\mathrm{p}=0.067$; $\mathrm{C}-3$ to $\mathrm{O}-1$ vs. $\mathrm{O}-2$ to $\mathrm{O}-3$ groups, $\mathrm{p}=0.083)$. When the number of detected cancers was examined in relation to the degree of atrophy, there were $3(0.02 \%)$ undifferentiated and $5(0.04 \%)$ differentiated cancer cases among 12,349 cases in the C- 0 to C-2 groups, $16(0.18 \%)$ undifferentiated and $47(0.54 \%)$ differentiated cancer cases among 8,644 cases in the C-3 to O-1 groups, and $32(0.47 \%)$ undifferentiated and 173 (2.60\%) differentiated cancer cases among 6,784 cases in the O-2 to O-3 groups (fig. 2). The number of both differentiated and undifferentiated cancers detected increased as the degree of gastric mucosal atrophy progressed (C-0 to $\mathrm{C}-2$ vs. $\mathrm{C}-3$ to $\mathrm{O}-1$ groups, $\mathrm{p}<0.001$; $\mathrm{C}-3$ to $\mathrm{O}-1$ vs. O-2 to $\mathrm{O}-3$ groups, $\mathrm{p}=0.002$ ).

Synchronous multiple cancers were found in 30 $(11.0 \%)$ of 272 patients with early gastric cancer. In relation to the degree of atrophy, there were no cases with synchronous multiple cancers in the $\mathrm{C}-0$ to $\mathrm{C}-2$ groups, 1 case in the C-3 group, 2 in the O-1 group, 14 in the O-2 group and 13 in the O-3 group (fig. 3). Of the 30 cases, 29
(96.7\%) were of the open type (closed type vs. open type, $\mathrm{p}<0.001)$. Metachronous multiple cancers were found in $20(7.4 \%)$ of 272 patients. In relation to the degree of atrophy, there were no cases with metachronous multiple cancers in the C- 0 to C- 3 groups, 2 cases in the O- 1 group, 7 in the O-2 group and 11 in the O-3 group (fig. 4). All 20 cases had open-type atrophy (closed type vs. open type, $\mathrm{p}<0.001)$.

\section{Discussion}

Individuals who have developed gastric cancer account for only a fraction of all $H$. pylori carriers. It is necessary to evaluate the gastric cancer risk and identify those at high risk for gastric cancer development among individuals who are positive for $\mathrm{H}$. pylori. Uemura et al. [6] evaluated endoscopic findings together with histological findings, and reported that severe atrophic gastritis (particularly corpus-dominant gastritis) accompanied by intestinal metaplasia represents a high risk for developing differentiated gastric cancer. In addition, Rugge et al. [10] attempted assessment of gastric cancer risk by evaluating the degree and distribution of atrophy and intestinal metaplasia using gastric biopsy specimens. In the present study, we focused on endoscopic atrophic gastritis that can be observed by a simple routinely performed method that does not require special devices, stains or gastric biopsy, and examined carcinogenic risk in relation to precise classifications of the degree of gastric mucosal atrophy.

When findings from all 27,777 cases were analyzed, the gastric cancer detection rate in relation to the degree of gastric mucosal atrophy was found to be $0.04 \%$ for C- 0 , $0 \%$ for C- $1,0.25 \%$ for $\mathrm{C}-2,0.71 \%$ for $\mathrm{C}-3,1.32 \%$ for $\mathrm{O}-1$, $3.70 \%$ for $\mathrm{O}-2$ and $5.33 \%$ for $\mathrm{O}-3$, showing a significant increase in the detection rate as atrophy progressed. $\mathrm{Pa}$ tient age also increased with the progression of gastric mucosal atrophy. These results support previous reports showing that the gastric carcinogenesis risk increases with the progression of atrophic changes with age [6]. According to the 2011 national registry issued by the Japanese Society of Gastrointestinal Cancer Screening, the gastric cancer detection rate in 453,951 individuals who underwent endoscopic screening was $0.28 \%$ [11]. Considering this figure, gastric cancer risk in association with C-0 and C- 1 atrophy is extremely low, while severe mucosal atrophy of C-3 or greater represents a high risk for developing gastric cancer. It is necessary to carry out endoscopic observation carefully, while keeping this finding 


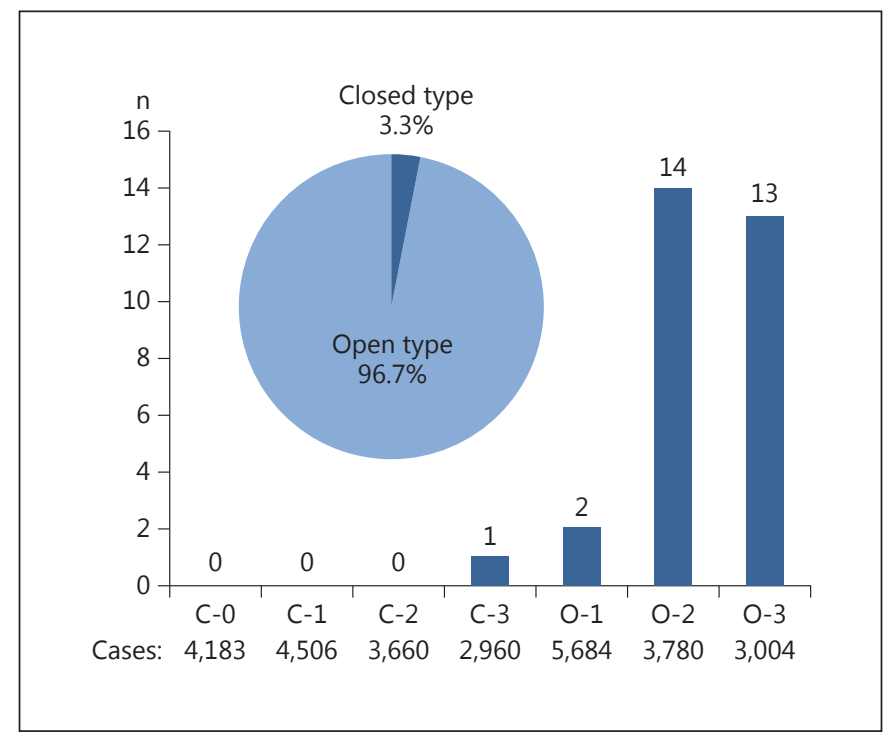

Fig. 3. The number of synchronous multiple gastric cancers in relation to the degree of gastric mucosal atrophy. Of the 30 cases, 29 (96.7\%) were of open-type atrophy.

in mind. It was inferred that the 2 patients found in the C-0 and C-1 groups had gastric cancer originating in $H$. pylori-negative gastric mucosa ( 1 patient was negative by the rapid urease test, serum $H$. pylori antibody and the urea breath test, and the other was negative for the rapid urease test, $H$. pylori antibody in urine and the pathological test). Matsuo et al. [12] reported the following characteristic features of $H$. pylori-negative gastric cancer: there is no difference in its incidence between men and women; it accounts for less than $1 \%$ of all gastric cancers; it occurs more frequently in younger patients, with the diffuse type being more frequent than in $H$. pylori-positive gastric cancer, and it has a concave shape. The 2 cases found in this study had features consistent with these characteristics of $H$. pylori-negative gastric cancer, except that one of the two had a differentiated gastric cancer.

Histological analysis of the early gastric cancers detected in this study revealed that both differentiated and undifferentiated tumors were more frequent in men than in women, and that in younger patients undifferentiated cancers were more frequent than differentiated cancers. As to the proportions of differentiated and undifferentiated cancers in relation to the degree of gastric mucosal atrophy, undifferentiated cancers were found to be more frequent when mucosal atrophy was absent or mild, but the frequency increased as mucosal atrophy progressed. This result is consistent with the carcinogenic sequence

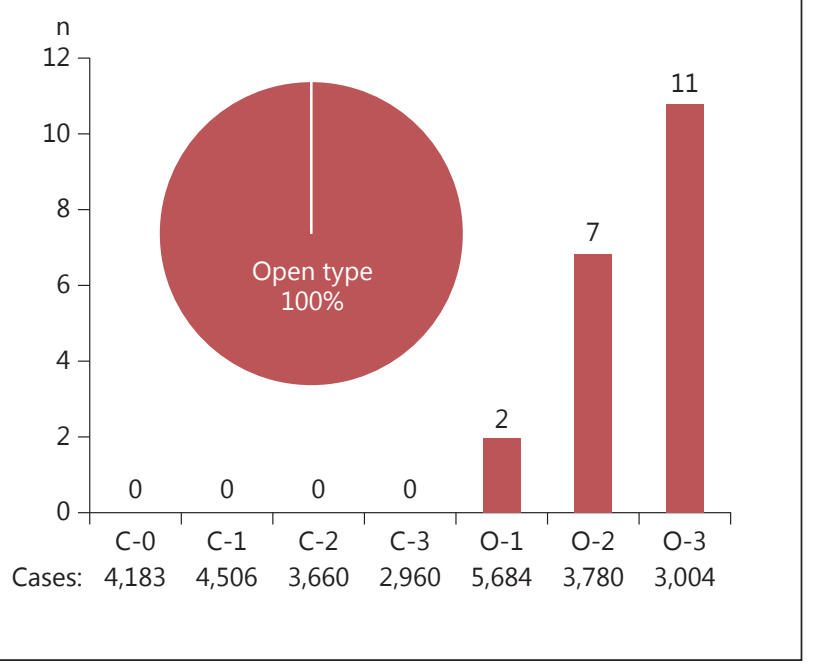

Fig. 4. The number of metachronous multiple gastric cancers in relation to the degree of gastric mucosal atrophy. All 20 cases had open-type atrophy.

in which atrophic gastritis advances into intestinal metaplasia and results in the occurrence of differentiated gastric cancer [13], and supports another report documenting that undifferentiated gastric cancer originates from the gastric mucosa not showing atrophy or intestinal metaplasia [14]. However, analysis of the total case population showed that the number of detected cancers increased as gastric mucosal atrophy progressed in both differentiated and undifferentiated cancer cases. Undifferentiated gastric cancer is reportedly not associated with atrophy or intestinal metaplasia, as mentioned previously. However, there are studies supporting our finding that undifferentiated gastric cancer also occurs in patients who have mucosal atrophy. It has been reported that gastritis with a goose bumps-like appearance and nodular gastritis associated with $H$. pylori infection represents a high risk for developing undifferentiated cancer [15-17]. Although the carcinogenic mechanisms underlying these forms of gastritis remain unclear, inflammation due to $H$. pylori infection is suspected to be involved. In addition, Uemura et al. [6] reported that undifferentiated cancer occurred frequently in cases with mild-to-moderate atrophy in the corpus with severe inflammation involving the gastric mucosa. Similar results were reported by Tanaka et al. [18], who examined the background mucosa in undifferentiated gastric cancer cases using gastric biopsy specimens. Tatemichi et al. [19] investigated gastric can- 
cer risk in terms of serum $H$. pylori antibody and pepsinogen levels, and reported that inflammation due to $H$. pylori infection was associated with undifferentiated gastric cancer. Based on these reports, it is likely that prolonged inflammation due to $H$. pylori infection led to the progression of gastric mucosal atrophy, resulting in the increased number of undifferentiated cancers detected.

In our present study, synchronous multiple cancers were found in $30(11.0 \%)$ patients, and metachronous multiple cancers in 20 (7.4\%); the frequencies were similar to those reported previously [20-22]. Interestingly, synchronous multiple cancers showed atrophy of the open type in all but 1 case, and all metachronous multiple cancers showed atrophy of the open type. These results suggest that patients with open-type atrophy are not only at high risk for gastric cancer, but are also at high risk for synchronous and metachronous multiple cancers, necessitating meticulous endoscopic follow-up. It was formerly believed, based on the results of studies by Wong et al. [23], that $H$. pylori eradication should be conducted prior to the development of changes such as atrophy and intestinal metaplasia to achieve a gastric cancer-preventing effect. Fukase et al. [24] carried out a prospective study of postendoscopic-treatment patients who were at high risk for gastric carcinogenesis, and demonstrated the incidence of metachronous gastric cancer to be decreased to about one third by $H$. pylori eradication over the course of 3 years after $H$. pylori eradication. The subjects of their study were a patient population who had moderate-tosevere atrophic mucosa accompanied by intestinal metaplasia, indicating that $H$. pylori eradication is effective even in patients who have advanced atrophy of the gastric mucosa. Based on long-term observation after $H$. pylori eradication, it has also been reported that eradicating this microorganism results in marked improvement of gastric mucosal atrophy and intestinal metaplasia, which are changes strongly related to the development of gastric cancer [25]. Therefore, it can reasonably be inferred that H. pylori eradication in cases with open-type atrophy is essential for preventing the occurrence of gastric cancer. In addition, long-term observation of patients who underwent $H$. pylori eradication reportedly revealed the occurrence of gastric cancer more than 10 years after eradication [26]. Therefore, long-term observation of the clinical course after $H$. pylori eradication is advisable.

It should be noted that our study has several limitations. Although the extent of gastric mucosal atrophy was evaluated, gastric cancer risk in relation to differences in the inflammation activity was not considered. Evaluation of atrophy is sometimes difficult and may depend on the skills of the examiner. Atypical atrophic changes were not addressed. Furthermore, this was a single-center study.

We conclude that endoscopic evaluation of gastric mucosal atrophy can provide a simple and reliable predictive index for current and future risks of gastric carcinogenesis. The results should be confirmed by future investigations using a multicenter prospective design.

\section{Disclosure Statement}

The authors declare that they have no competing interests.

\section{References}

1 Correa P: The gastric precancerous process. Cancer Survey 1983;2:437-450.

- 2 Imai T, Kubo T, Watanabe H: Chronic gastritis in Japanese with reference to high incidence of gastric carcinoma. J Natl Cancer Inst 1971;47:179-195.

3 Asaka M, Sugiyama T, Nobuta A, Kato M, Takeda H, Graham DY: Atrophic gastritis and intestinal metaplasia in Japan: results of a large multicenter study. Helicobacter 2001;6: 294-299.

-4 Parsonnet J, Friedman GD, Vandersteen DP, Chang Y, Vogelman JH, Orentreich N, Sibley RK: Helicobacter pylori infection and the risk of gastric carcinoma. N Engl J Med 1991;325: 1127-1131.

5 Nomura A, Stemmermann GN, Chyou PH, Kato I, Perez-Perez GI, Blaser MJ: Helicobacter pylori infection and gastric carcinoma among Japanese Americans in Hawaii. N Engl J Med 1991;325:1132-1136.

-6 Uemura N, Okamoto S, Yamamoto S, Matsumura N, Yamaguchi S, Yamakido M, Taniyama K, Sasaki N, Schlemper RJ: Helicobacter pylori infection and the development of gastric cancer. N Engl J Med 2001;345:784-789.

7 Kimura K, Takemoto T: An endoscopic recognition of the atrophic border and its significance in chronic gastritis. Endoscopy 1969;3: 87-97.

-8 Japanese Gastric Cancer Association: Japanese Classification of Gastric Carcinoma, English ed 3. Gastric Cancer 2011;14:101-112.

-9 Lauren P: The two histological main types of gastric carcinoma: diffuse and so-called intestinal-type carcinoma: an attempt at a histoclinical classification. Acta Pathol Microbiol Scad 1965;64:31-49.
10 Rugge M, de Boni M, Pennelli G, de Bona M, Giacomelli L, Fassan M, Basso D, Plebani M, Graham DY: Gastritis OLGA-staging and gastric cancer risk: a twelve-year clinico-pathological follow-up study. Aliment Pharmacol Ther 2010;31:1104-1111.

11 Japanese Society of Gastrointestinal Cancer Screening: Annual report of gastrointestinal cancer screening in Japan, 2011. J Gastroenterol Cancer Screen 2014;52:70-96.

12 Matsuo T, Ito M, Takata S, Tanaka S, Yoshihara M, Chayama K: Low prevalence of Helicobacter pylori-negative gastric cancer among Japanese. Helicobacter 2011;16:415-419.

13 Kuipers EJ, Uyterlinde AM, Peña AS, Roosendaal R, Pals G, Nelis GF, Festen HP, Meuwissen SG: Long-term sequelae of Helicobacter pylori gastritis. Lancet 1995;345:15251528.
Degree of Endoscopic Atrophy of Gastric Mucosa and Carcinogenic Risk 
14 Vauhkonen M, Vauhkonen H, Sipponen P: Pathology and molecular biology of gastric cancer. Best Pract Res Clin Gastroenterol 2006;20:651-674

15 Haruma K, Komoto K, Kamada T, Ito M, Kitadai Y, Yoshihara M, Sumii K, Kajiyama G: Helicobacter pylori infection is a major risk factor for gastric carcinoma in young patients. Scand J Gastroenterol 2000;35:255-259.

16 Kamada T, Hata J, Tanaka A, Kusunoki H, Miyamoto M, Inoue K, Sadahira Y, Haruma K: Nodular gastritis and gastric cancer. Dig Endosc 2006;18:79-83.

17 Nishibayashi H, Kanayama S, Kiyohara T, Yamamoto K, Miyazaki Y, Yasunaga Y, Shinomura Y, Takeshita T, Takeuchi T, Morimoto $\mathrm{K}$, Matsuzawa Y: Helicobacter pylori-induced enlarged-fold gastritis is associated with increased mutagenicity of gastric juice, increased oxidative DNA damage, and an increased risk of gastric carcinoma. J Gastroenterol Hepatol 2003;18:1384-1391.

18 Tanaka A, Kamada T, Inoue K, Shiotani A, Kusunoki H, Manabe N, Ito M, Hata J, Haruma K: Histological evaluation of patients with gastritis at high risk of developing gastric cancer using a conventional index. Pathol Res Pract 2011;207:354-358.
19 Tatemichi M, Sasazuki S, Inoue M, Tsugane S: Different etiological role of Helicobacter pylori $(H p)$ infection in carcinogenesis between differentiated and undifferentiated gastric cancers: a nested case-control study using IgG titer against $H p$ surface antigen. Acta Oncologica 2008;47:360-365.

20 Nakajima T, Oda I, Gotoda T, Hamanaka H, Eguchi T, Yokoi C, Saito D: Metachronous gastric cancers after endoscopic resection: how effective is annual endoscopic surveillance? Gastric Cancer 2006;9:93-98.

21 Isobe T, Hashimoto K, Kizaki J, Murakami N, Aoyagi K, Koufuji K, Akagi Y, Shirouzu K: Characteristics and prognosis of synchronous multiple early gastric cancer. World J Gastroenterol 2013;19:7154-7159.

22 Shiotani A, Uedo N, Iishi H, Yoshiyuki Y, Ishii M, Manabe N, Kamada T, Kusunoki H, Hata J, Haruma K: Predictive factors for metachronous gastric cancer in high-risk patients after successful Helicobacter pylori eradication. Digestion 2008;78:113-119.
23 Wong BC, Lam SK, Wong WM, Chen JS, Zheng TT, Feng RE, Lai KC, Hu WH, Yuen ST, Leung SY, Fong DY, Ho J, Ching CK, Chen JS: Helicobacter pylori eradication to prevent gastric cancer in a high-risk region of China: a randomized controlled trial. JAMA 2004;291:187-194.

24 Fukase K, Kato M, Kikuchi S, Inoue K, Uemura N, Okamoto S, Terao S, Amagai K, Hayashi S, Asaka M: Effect of eradication of Helicobacter pylori on incidence of metachronous gastric carcinoma after endoscopic resection of early gastric cancer: an open-label, randomised controlled trial. Lancet 2008;372: 392-397.

25 Kodama M, Murakami K, Okimoto T, Abe T, Nakagawa Y, Mizukami K, Uchida M, Inoue $\mathrm{K}$, Fujioka T: Helicobacter pylori eradication improves gastric atrophy and intestinal metaplasia in long-term observation. Digestion 2012;85:126-130.

26 Take S, Mizuno M, Ishiki K, Yoshida T, Ohara N, Yokota K, Oguma K, Okada H, Yamamoto K: The long-term risk of gastric cancer after the successful eradication of Helicobacter pylori. J Gastroenterol 2011;46:318324 